\title{
PROCESOS DE FOTOCATÁLISIS SOLAR PARA LA DETOXIFICACIÓN Y DESCONTAMINACIÓN DE AGUAS
}

\author{
SOLAR PHOTOCATALYSIS APPLIED FOR \\ WATER DETOXIFICATION \\ AND DECONTAMINATION
}

\author{
Pilar Fernández Ibáñez ${ }^{1}$
}

RESUMEN

\begin{abstract}
Una de las características que mejor define la sociedad actual es la producción de residuos, siendo esta superior en los denominados países "desarrollados". Prácticamente no hay actividad humana alguna que no genere residuos existiendo además una relación directa entre el nivel de vida de una sociedad o país y la cantidad de residuos generados. Aproximadamente, el $23 \%$ de la población mundial vive en países desarrollados, consume el $78 \%$ de los recursos y produce el $82 \%$ de los residuos [Blanco y Malato, 1996]. Además, el volumen de residuos crece de forma exponencial con el nivel de industrialización de un país. Existen actualmente registradas unos cinco millones de sustancias conocidas de las que aproximadamente 70.000 son ampliamente usadas en todo el mundo, estimándose en unas 1.000 las nuevas sustancias químicas que cada año son incorporadas a la lista.
\end{abstract}

Palabras clave.- Fotocatálisis, Descontaminación de aguas.

\begin{abstract}
One characteristic that best defines today's society is the production of waste, this being higher in so-called "developed" countries. Virtually no human activity that does not generate any waste also exist a direct relationship between the standard of living of a society or country and the amount of waste generated. Approximately 23\% of the world population lives in developed countries consume $78 \%$ of resources and produces $82 \%$ of the waste [Black and Malate, 1996]. Furthermore, the volume of waste grows exponentially with the level of industrialization of a country. There are currently about five million registered substances known of which approximately 70,000 are widely used throughout the world, estimated at about 1,000 new chemicals each year are added to the list.
\end{abstract}

Keywords.- Photocatalysis, Water decontamination.

\section{INTRODUCCIÓN \\ PROBLEMÁTICA DE LA CONTAMINACIÓN DE LAS AGUAS}

La necesidad de un crecimiento sostenible se contrapone con la realidad del crecimiento demográfico. Muchos países atraviesan una etapa de crecimiento no-sostenible, con logros macroeconómicos muy variables en los distintos países. Aún en los casos en los que los países han alcanzado interesantes progresos macroeconómicos, estos logros no se reflejan en la calidad de vida de grandes porciones de la población. Esos grandes contrastes se reflejan en los problemas asociados con la gestión racional

${ }^{1}$ Centro de investigaciones energéticas, medioambientales y tecnológicas (CIEMAT), Plataforma solar de Almeria (PSA), Tabernas, Almeria. 
del agua, que no puede enfrentarse de forma unilateral, si no que hay muchos procedimientos.

Una publicación reciente [Schertenleib y Gujer, 2000] describe de forma genérica los problemas que han debido enfrentar las sociedades en función del uso del agua, a medida que dicha sociedad iba evolucionando. Los países de desarrollo sostenible han ido enfrentando uno por uno, problemas relacionados con la contaminación biológica, con los niveles de metales pesados, con el uso intensivo de nutrientes, y con los contaminantes orgánicos a niveles de ultra-traza. La desinfección de aguas, el tratamiento de efluentes antes de su vertido a cauces de agua, la limitación y sustitución de nitratos y fosfatos en productos de uso masivo, y los desarrollos en química analítica y en ecotoxicología ilustran algunas de las herramientas usadas para enfrentar dichas categorías de problemas.

El principal problema ambiental lo constituyen los vertidos procedentes de la industria y la agricultura, aunque la población también juega un papel destacado en la contaminación del medio ambiente. Plaguicidas, fertilizantes, detergentes, fenoles y otros productos químicos son arrojados sin tratamiento directamente a la naturaleza, a través de vertederos, controlados o incontrolados y situados sin una estrategia de tratamiento.

Una gran parte de este tipo de residuos son generados en solución acuosa y, debido a su propia naturaleza de no biodegradabilidad, los procesos de tratamiento biológico (los más comúnmente utilizados) no tienen ninguna acción sobre ellos por lo que, si no hay ningún tratamiento específico adicional, acaban vertidos sobre el medio ambiente. La presencia de este tipo de contaminantes en disolución acuosa resulta especialmente problemática ya que el residuo no puede ser almacenado indefinidamente (como ocurre con algunos residuos sólidos) y tiene la particularidad de que pequeños volúmenes de agua pueden contaminar, a su vez, otros volúmenes de agua mucho mayores.

\section{Técnicas de tratamientos de aguas}

El objetivo de cualquier tratamiento de contaminantes en agua es eliminar ciertos contaminantes, normalmente mezclas complejas de compuestos orgánicos e inorgánicos, presentes en un efluente de tal manera que una vez tratado cumpla las especificaciones de vertido determinadas por el organismo competente. Para tratar un determinado efluente, se ha de realizar una caracterización del mismo: de sus materias contaminantes, de su caudal, así como de las oscilaciones que en ambos factores puedan producirse. En función de dichos parámetros, las técnicas de tratamiento se estructuran como en la figura 1. Cuando se trata de eliminar compuestos muy específicos y difíciles de degradar por los métodos expuestos, se utilizan los procesos abajo indicado.

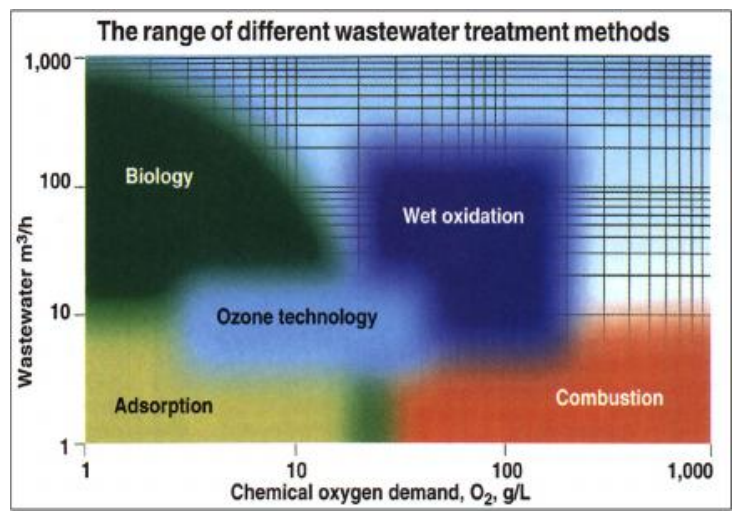

Fig. 1 Técnicas de tratamiento de aguas.

\section{Procesos de oxidación avanzada}

Los Procesos de Oxidación Avanzada son aquellos procesos de tratamiento basados en la generación de especies fuertemente oxidantes [Andreozzi y col., 1999]. Entre ellos, los que producen radicales hidroxilos $\left({ }^{\circ} \mathrm{OH}\right)$ son los que tienen más capacidad depuradora, ya que esta especie es un agente oxidante muy enérgico, siendo capaz de oxidar una amplia variedad de compuestos orgánicos. En resumen, las ventajas más importantes de este tipo de procesos son las siguientes:

- Capacidad para llevar a cabo una profunda mineralización de los contaminantes orgánicos y oxidación de compuestos inorgánicos hasta dióxido de carbono e iones inorgánicos.

- Reactividad no selectiva con una inmensa mayoría de compuestos orgánicos. Hecho especialmente interesante si se quiere evitar la presencia de subproductos potencialmente tóxicos procedentes de los 
contaminantes originales, que sí pueden originarse mediante otros métodos que no consiguen la oxidación hasta sus últimas consecuencias.

El inconveniente fundamental de los mismos es su elevado coste por el uso de reactivos caros (p.e. $\mathrm{H}_{2} \mathrm{O}_{2}$ ) y/o el elevado consumo energético (lámparas para generar radiación UV) y es obvio que nunca deben utilizarse como alternativa a tratamientos más económicos, como la biodegradación.

\section{Procesos fotocatalíticos basados en la luz solar}

De entre todos los AOPs aquellos que son capaces de aprovechar la radiación solar son de especial interés, ya que se eliminaría la desventaja que tienen estos procesos del consumo de energía mediante lámparas, ya que éstas son sustituidas por el Sol. Estos procesos son la fotocatálisis "heterogénea" con $\mathrm{TiO}_{2}$ y fotocatálisis "homogénea" con foto-Fenton.

La descontaminación de aguas contaminadas mediante fotocatálisis solar es en la actualidad una de las más exitosas aplicaciones de la fotoquímica solar [Bahnemann y col., 1993, 1994a y 1994b; Bauer, 1994 y 1999; Blanco y Malato, 2001; Bockelmann y col., 1995; Curcó y col., 1997; Freundenhammer y col., 1997; Goslich R. y col., 1997; Giménez y col., 1997 y 1999; Herrmann y col., 1998 y 1999; Malato y col., 1999 y 2002; Marques y col., 1996; Minero, 1997; Zhang y col., 2001]. Esto no es sólo debido a que es una excepcional demostración de la aplicación de la energía solar a la conservación medioambiental, sino que, al contrario de la mayoría de los procesos fotoquímicos, su versatilidad permite que sea empleada con mezclas complejas de contaminantes.

Se define la fotocatálisis solar como la aceleración de una foto reacción química mediante la presencia de un catalizador [Mills y Le Hunte, 1997]. Tanto la luz como el catalizador son elementos necesarios. El término fotocatálisis incluye un proceso según el cual se produce una alteración fotoquímica de alguna especie química como resultado de la absorción de radiación por otra especie fotosensible, que en este caso se trata del catalizador. El concepto de fotocatálisis heterogénea es simple: el uso de un sólido semiconductor (ej.: $\mathrm{TiO}_{2}, \mathrm{ZnO}$, etc.) que forma una suspensión estable bajo irradiación para estimular una reacción en la interfase sólido / líquido o sólido / gas. En el caso de la fotocatálisis homogénea el catalizador está disuelto en el agua que contiene los contaminantes a tratar, también disueltos, por lo que todos los elementos que intervienen en el proceso se encuentran en la misma fase. Por definición, en ambos casos, el catalizador debe de poder ser reutilizado después de actuar en el sistema de oxidación-reducción sin sufrir cambios significativos.

\section{Fotocatálisis heterogénea con $\mathrm{TiO}_{2}$}

En el caso de los procesos donde interviene un sólido semiconductor, el mecanismo de fotorreacción (Ecs. 1, 2 y 3) comienza cuando un semiconductor del tipo calcogénico (óxidos como $\mathrm{TiO}_{2}, \mathrm{ZnO}, \mathrm{ZrO}_{2}, \mathrm{CeO}_{2}$, etc., o sulfuros como CdS, $\mathrm{ZnS}$, etc.) es iluminado con fotones cuya energía es igual o mayor a la separación energética existente entre la banda de valencia y la de conducción. En esta situación, tiene lugar una absorción de esos fotones y la creación en el seno del catalizador de pares electrón-hueco $\left(\mathrm{e}^{-} \mathrm{y} \mathrm{h}^{+}\right)$, los cuales se disocian en foto-electrones libres en la banda de conducción y foto-huecos en la banda de valencia. Simultáneamente, tiene lugar una adsorción de reactivos $\mathrm{y}$, de acuerdo con el potencial redox (o nivel de energía) del catalizador, un electrón es transferido hacia una molécula aceptora $\left(\mathrm{Ox}_{1}\right)$, produciendo una reacción de reducción de la misma; al mismo tiempo, un foto-hueco es transferido hacia una molécula donadora $\left(\operatorname{Red}_{2}\right)$, que se oxidará. Cada ión formado reacciona para formar productos intermedios y finales. El flujo neto de electrones es nulo y el catalizador permanece inalterado. La excitación fotónica del catalizador aparece como el paso inicial de la activación de la totalidad del sistema catalítico.

$$
\begin{gathered}
C \stackrel{h v}{\longrightarrow} C\left(e^{-}+h^{+}\right) \\
h^{+}+\operatorname{Re} d_{2} \longrightarrow O x_{2} \\
e^{-}+O x_{1} \longrightarrow \operatorname{Re} d_{1}
\end{gathered}
$$

Multitud de sustancias semiconductoras han sido probadas para la degradación de compuestos. Sin embargo, el fotocatalizador con el que se han obtenido mejores resultados es el dióxido de 
titanio $\left(\mathrm{TiO}_{2}\right)$ [Andreozzi y col., 1999; Blake D.M., 1994, 1995, 1997, 1999 y 2001; Guillard y col., 1999; Hermann y col., 1999a y 1999d; Prousek, 1996].

El proceso fotocatalítico global puede dividirse en 5 pasos independientes:

1. Transferencia del reactivo de la fase fluida a la superficie del catalizador.

2. Adsorción de al menos uno de los reactivos.

3. Reacción en la fase adsorbida bajo irradiación (reacción fotocatalítica).

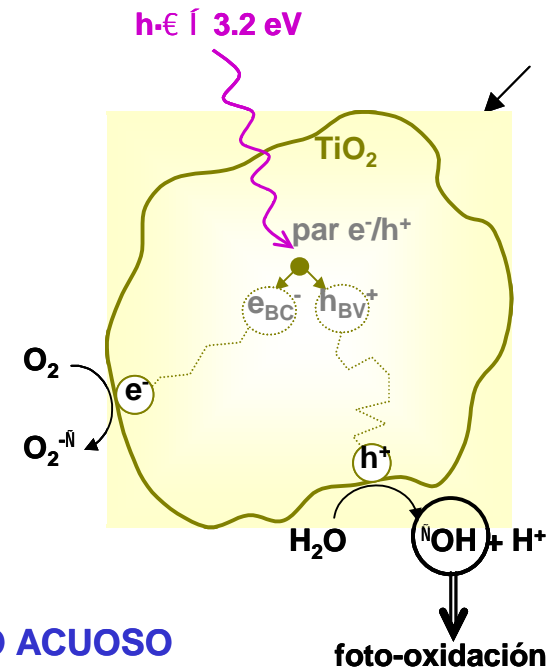

\section{MEDIO ACUOSO}

4. Desorción de los productos.

5. Transferencia de los productos a la fase fluida.

El proceso fotocatalítico heterogéneo mediante $\mathrm{TiO}_{2}$ se muestra en la figura 2, donde la luz (que puede ser tanto solar como artificial) con fotones de energía superior al ancho de banda del semiconductor promueve el paso de un electrón desde la banda de valencia hasta la banda de conducción para acabar generando, en presencia de agua, radicales hidroxilo $\left({ }^{\circ} \mathrm{OH}\right)$.

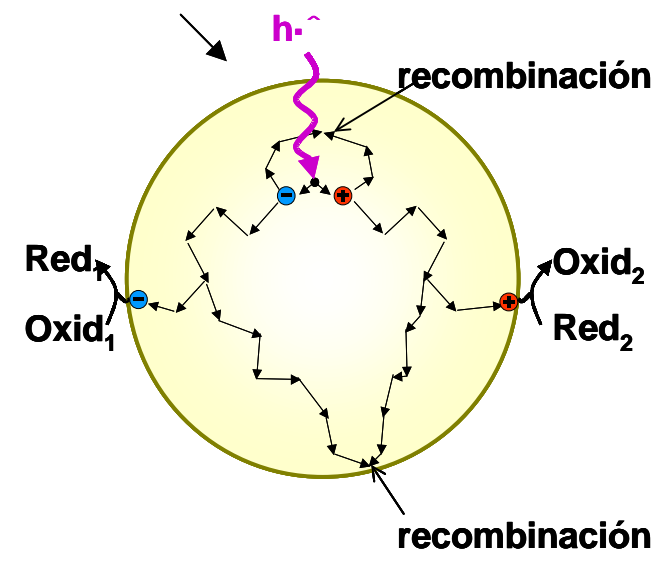

Fig. 2 Representación de proceso fotocatalítico heterogéneo en una partícula de catalizador $\mathrm{TiO}_{2}$, a la derecha recombinación de pares $e^{-} / h^{+}$.

La formación de los distintos radicales, así como otros procesos que pueden tener lugar durante la fotocatálisis mediante el uso de $\mathrm{TiO}_{2}$, se pueden explicar mediante las reacciones 4 a 13 [Serpone y col., 1993]. La primera consecuencia, tras la absorción de radiación (UV cercano, $\lambda \leq 387 \mathrm{~nm}$ ), es la generación de pares $\mathrm{e}^{-} / \mathrm{h}^{+}$que se separan entre la banda de valencia y la de conducción. Ambos migran rápidamente hacia la superficie, donde el $\mathrm{h}^{+}$es atrapado por trampas de energía subsuperficial y el e $\mathrm{e}^{-}$por trampas superficiales, o bien por trampas externas mediante donantes de electrones $\left(\operatorname{Red}_{2, \mathrm{ads}}\right)$ y captadores de electrones $\left(\mathrm{Ox}_{1, \mathrm{ads}}\right)$, respectivamente. Para impedir la recombinación de los pares generados (ec. 5), se requiere que $\operatorname{Red}_{2}$ y $\mathrm{Ox}_{1}$ estén previamente adsorbidos en la superficie, antes de la excitación del catalizador por la radiación. Si el disolvente es oxidorreductoramente activo (agua) también actúa como donante y aceptor de electrones, de manera que para una superficie de $\mathrm{TiO}_{2}$ hidratada e hidroxilada, los huecos atrapados generan radicales ${ }^{\circ} \mathrm{OH}$ enlazados a la superficie. No obstante, los electrones y huecos atrapados pueden recombinarse rápidamente en la superficie de la partícula. Para prevenir este proceso el electrón puede ser capturado por oxígeno molecular preadsorbido formando el radical superóxido $\mathrm{O}_{2}{ }^{\circ}$. En fase acuosa y en presencia de oxígeno disuelto, el agua actúa como elemento reductor. Como consecuencia, el agua y oxígeno resultan elementos esenciales en el proceso, no existiendo degradación alguna en su ausencia. 


$$
\begin{aligned}
& \mathrm{TiO}_{2}+h \mathrm{v} \stackrel{h v \geq E_{G}}{\longrightarrow} e_{B C}^{-}+h_{B V}^{+} \\
& e_{B C}^{-}+h_{B V}^{+} \rightarrow \mathrm{TiO}_{2}+\text { calor y/o hv } \\
& \left(\mathrm{TiO}^{I V}-\mathrm{O}^{2-}-\mathrm{Ti}^{I V}\right)_{\text {subsuperfcie }}+h_{B V}^{+} \rightarrow\left(\mathrm{TiO}^{I V}-\mathrm{O}^{\bullet-}-\mathrm{Ti}^{I V}\right)_{\text {subsuperfcie }} \\
& \left(-T i^{I V}-\right)_{\text {superficie }}+e_{B C}^{-} \rightarrow\left(-T i^{I I I}-\right)_{\text {superficie }} \\
& h_{B V}^{+}+\operatorname{Re} d_{2, a d s} \rightarrow O x_{2, a d s} \\
& e_{B C}^{-}+O x_{1, a d s} \rightarrow \operatorname{Re} d_{1, a d s} \\
& \left(\mathrm{TiO}^{I V}-\mathrm{O}^{2-}-\mathrm{Ti}^{I V}\right)-\mathrm{OH}^{-}+h_{B V}^{+} \leftrightarrow\left(\mathrm{TiO}^{I V}-\mathrm{O}^{2-}-\mathrm{Ti}^{I V}\right)--^{\cdot} \mathrm{OH} \\
& \left(\mathrm{TiO}^{\mathrm{IV}}-\mathrm{O}^{2-}-\mathrm{Ti}^{I V}\right)-\mathrm{OH}_{2}+h_{\mathrm{BV}}^{+} \leftrightarrow\left(\mathrm{TiO}^{\mathrm{IV}}-\mathrm{O}^{2-}-\mathrm{Ti}^{I V}\right)-\mathrm{OH}^{\bullet}+\mathrm{H}^{+} \\
& \mathrm{O}_{2(\text { ads })}+e^{-} \rightarrow \mathrm{O}_{2(\text { ads })}^{\bullet-} \\
& \mathrm{H}_{2} \mathrm{O}+h_{B V}^{+} \rightarrow{ }^{\bullet} \mathrm{OH}+\mathrm{H}_{a q}^{+}
\end{aligned}
$$

Las evidencias han demostrado que los radicales - $\mathrm{OH}$ son los principales causantes de las reacciones de oxidación de contaminantes orgánicos mediante la ruptura progresiva de sus enlaces. Se ha demostrado la descomposición total de compuestos orgánicos simples y complejos (halogenados, derivados de alcanos, alquenos, ácidos carboxílicos y sustancias aromáticas, etc) mediante fotocatálisis heterogénea.

En compuestos aromáticos, por ejemplo, se produce la hidroxilación de la parte aromática y sucesivos pasos de oxidación / adición llevan a la apertura del anillo. Los aldehídos y ácidos carboxílicos resultantes son descarboxilados y finalmente producen $\mathrm{CO}_{2}$. Hay que tener en cuenta que, al tratarse de un proceso de oxidaciónreducción, se puede usar también la vía reductiva (ver figura 1.2.4).

Un ejemplo puede ser la reducción mediante fotocatálisis de metales (como es el caso de $\mathrm{Cr}^{6+}$ a $\left.\mathrm{Cr}^{3+}\right)$, de forma simultánea a la oxidación de compuestos orgánicos [Aguado y col., 1991; Prairie y col., 1992 y 1994].

\section{Radiación Solar}

Debido a que una de las claves de todo proceso fotocatalítico, tanto heterogéneo como homogéneo, es la existencia de una fuente de radiación ultravioleta, es de especial interés la radiación solar, tanto de forma general como en su componente UV, que es la que más interesa en este caso, en particular el rango donde el catalizador puede ser fotoexcitado (Fig. 3).

Del total de la energía asociada a la radiación solar, considerando el espectro estándar normalizado a $1000 \mathrm{~W} / \mathrm{m}^{2}$, el $7,82 \%$ de dicha radiación es debido a radiación UV (longitud de onda $\leq 400 \mathrm{~nm}$ ); el 47,33\% corresponde a la luz visible, y el $44,85 \%$ restante es debido a la radiación infrarroja (longitud de onda $\geq 700 \mathrm{~nm}$ ) [Hulstrom y col., 1985]. 


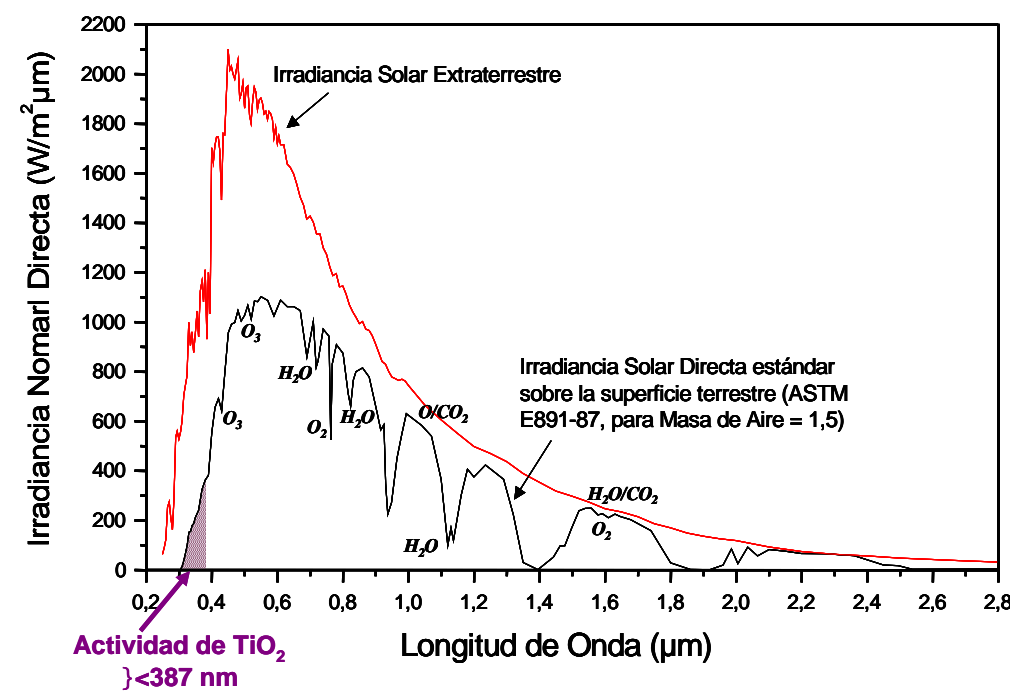

Fig. 3 Espectro de radiación solar sobre la superficie terrestre.

\section{Factores que afectan a la fotocatálisis con $\mathrm{TiO}_{2}$}

Los parámetros que afectan a los procesos fotocatalíticos en fase heterogénea, fundamentalmente son el oxígeno disuelto, el $\mathrm{pH}$ del medio, la concentración de catalizador, la concentración inicial de sustrato, la intensidad de radiación y la temperatura a la que tienen lugar los procesos de fotocatálisis [Blanco y Malato, 2001].

Aunque los contaminantes orgánicos suelen absorber radiación en un amplio rango de longitudes de onda, como norma general su absorción suele ser más fuerte a longitudes de onda cortas. Esta fotodegradación suele ser muy débil y lenta [Tomlin, 1994] en comparación con su degradación fotocatalítica [Blanco y Malato, 2001]. Además de estos parámetros, es necesario evaluar una serie de procesos que pueden ocurrir durante el tratamiento fotocatalítico y que pueden falsear los resultados. En cualquier caso, es importante tener en cuenta este fenómeno y se deben hacer ensayos de descomposición de los compuestos objeto de estudio en ausencia de catalizador con el fin de atribuir a la acción del catalizador sólo la parte que le corresponde. Otro tipo de experimentos están destinados a comprobar o evaluar la importancia de efectos como que el aumento de temperatura en el foto-reactor no provoque pérdidas del producto a degradar por evaporación o termolisis. Además, es necesario garantizar la ausencia de adsorción de las moléculas de contaminante en los componentes y materiales del foto-reactor. Cualquiera de estos efectos secundarios, que puedan darse durante los experimentos de fotocatálisis solar heterogénea en un foto-reactor, pueden cuantificarse y restarse del proceso global para conocer la eficiencia real de la reacción fotocatalítica.

\section{Oxígeno disuelto}

La fotocatálisis aplicada al tratamiento de aguas suele aplicarse a moléculas orgánicas, por lo que el proceso en su totalidad puede describirse según la ecuación 14. Por ello, no se producirá fotomineralización del contaminante en ausencia de oxígeno. Además, el oxígeno no compite con las moléculas de contaminante durante la adsorción sobre el $\mathrm{TiO}_{2}$, ya que los lugares superficiales donde se producen las reacciones de oxidación y reducción son distintos [Gerischer y Heller, 1992; Pelizzetti y col., 1990].

$$
\text { Orgánico }+\mathrm{O}_{2} \underset{\text { Semiconductor \& Rad-UV }}{\longrightarrow} \mathrm{CO}_{2}+\mathrm{H}_{2} \mathrm{O}+\text { ácidos inorgánicos }
$$


El $\mathrm{O}_{2}$ es altamente electrofílico, por lo que capta los electrones liberados tras la separación de pares y evita su recombinación generando así el radical superóxido $\left(\mathrm{O}_{2}{ }^{-\cdots}\right)$ [Pelizzetti, 1995].

\section{pH del medio}

El pH del medio acuoso afecta de forma significativa al $\mathrm{TiO}_{2}$ debido a que, como ya se ha explicado anteriormente, los iones determinantes de potencial del semiconductor son los $\mathrm{H}^{+}$y $\mathrm{OH}^{-}$. Por lo que una modificación del $\mathrm{pH}$ induce cambios en la carga eléctrica superficial y en el estado de agregación de las partículas de $\mathrm{TiO}_{2}$. El punto de carga nula del dióxido de titanio Degussa P25 es pH 7 [Fernández-Ibáñez et al., 2003]; por lo que para valores de $\mathrm{pH}$ inferiores las partículas presentan carga positiva y para valores superiores negativa. En muchos casos, este factor tan importante de los procesos fotocatalíticos no se controla debido, fundamentalmente, a que durante el tratamiento de agua contaminada aparecen una serie de compuestos intermedios y productos finales de la degradación que provocan cambios en el $\mathrm{pH}$ y cuyo comportamiento además depende del mismo.

Por otro lado, el tamaño medio de los agregados de partículas de catalizador depende altamente del $\mathrm{pH}$ del medio que los rodea. A valores de $\mathrm{pH}$ suficientemente alejados del punto de carga nula las partículas de $\mathrm{TiO}_{2}-\mathrm{P} 25$ conforman agregados de tamaño medio de unos 320-340 nm. En las proximidades del punto de carga nula las interacciones eléctricas desaparecen por la ausencia de carga superficial y la agregación entre partículas aumenta hasta diámetros de 2-4 micras. Estos cambios de tamaño de partícula (o agregado de partículas) evidentemente producirán una influencia importante sobre la eficiencia de los procesos de fotocatálisis, ya que el comportamiento de la transmisión, dispersión y absorción de radiación cambia en función del tamaño y de la concentración de las partículas. Además, el tamaño medio de partícula determina que la suspensión ofrezca una mayor o menor superficie en contacto con el medio acuoso. De este modo, una suspensión de partículas muy pequeñas presenta una superficie de catalizador muy superior a la que exponen partículas o agregados de mayor diámetro.
Adicionalmente, las partículas más grandes tienden a sedimentar más rápidamente que las pequeñas, por lo que es necesaria la aplicación de agitación mecánica a las suspensiones que garantice la homogeneidad de la misma. Sin embargo, esto supone una ventaja cuando, una vez finalizado el proceso de fotocatálisis, se debe separar el catalizador del agua mediante sedimentación y filtración para completar el proceso de tratamiento del agua.

\section{Concentración de catalizador}

Habitualmente, las velocidades de degradación son directamente proporcionales a la cantidad de catalizador empleado. Sin embargo, por encima de ciertas cantidades de catalizador (alrededor de 100 $\mathrm{mg} / \mathrm{L}$ ), la velocidad de reacción resulta ser independiente de la masa de catalizador (Fig. 4). Este límite depende de la geometría y de las condiciones de trabajo del reactor, y coincide con la concentración para la cual todas las partículas de $\mathrm{TiO}_{2}$ son correctamente iluminadas. Cuando la concentración de partículas está por encima de ese límite, la turbidez de la suspensión sólo permite que la radiación penetre una distancia (paso óptico) inferior a la profundidad del reactor, por lo que cierta cantidad de catalizador, en el interior del foto-reactor, queda sin iluminar y no efectúa su función catalizadora. Y si la concentración de catalizador es menor que la óptima, cierta proporción de la radiación incidente saldrá del foto-reactor sin ser absorbida por las partículas semiconductoras.

Existe una gran cantidad de publicaciones que estudian el efecto de la concentración de catalizador sobre la eficiencia del proceso. De todas ellas se deduce que es fundamental evaluar la radiación incidente y longitud de paso óptico del foto-reactor para determinar la concentración óptima de catalizador. En particular, cuando la fuente de radiación es externa al foto-reactor y éste tiene una longitud de paso óptico del orden de varios centímetros, la concentración óptima de $\mathrm{TiO}_{2}$ es de varios cientos de miligramos por litro [Blanco y Malato, 2001]. 


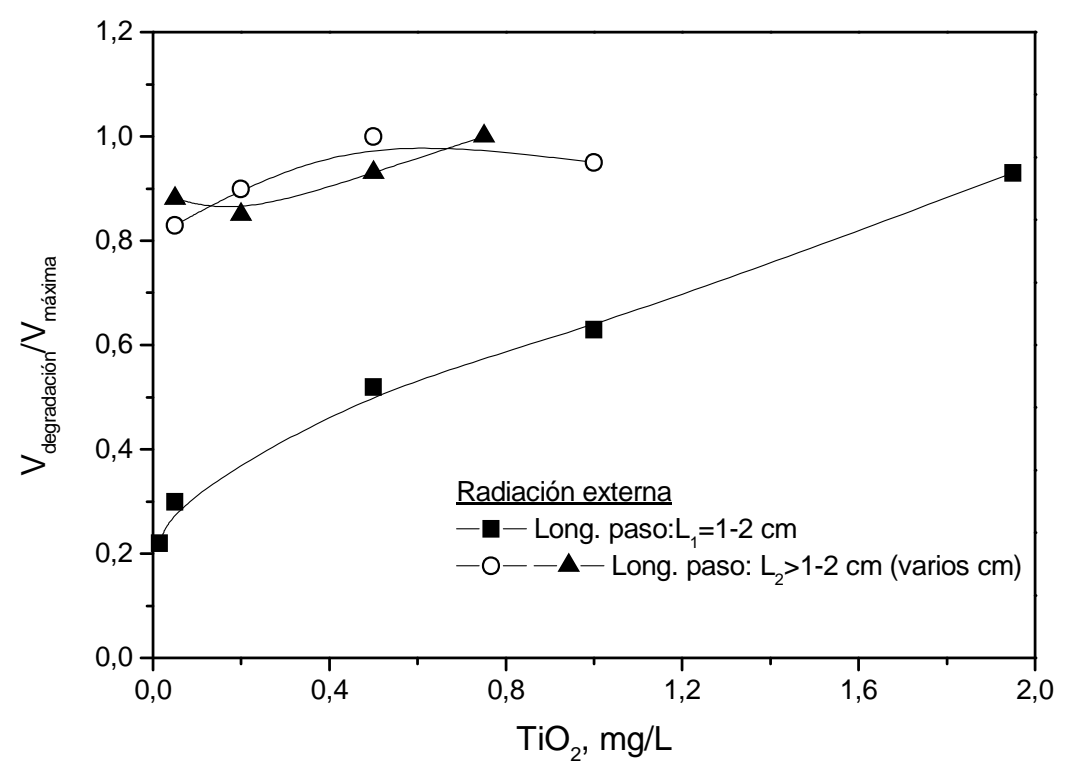

Fig. 4 Velocidad de degradación normalizada en dos reactores distintos con fuentes de radiación externa. Diámetro de 1-2 cm (símbolos cuadrados). Diámetro de varios cm (símbolos de círculos y triángulos). Gráfica tomada de [Blanco y Malato, 2001].

\section{Concentración inicial de contaminante}

Cuando tiene lugar el proceso de fotocatálisis de un contaminante, la cantidad de moléculas adsorbidas sobre la superficie del catalizador tiende a ser cada vez menor. La mayoría de los autores coinciden en indicar que la velocidad de reacción viene dada por la ley de LangmuirHinshelwood (L-H). Utilizando el modelo L-H, ecuación 15 y su linealizada 16, se obtienen las gráficas de la figura 5 .

$$
\begin{aligned}
& r=-\frac{d C}{d t}=\frac{k_{r} K C}{1+K C} \\
& \frac{1}{r}=\frac{1}{k_{r} K} \frac{1}{C}+\frac{1}{k_{r}}
\end{aligned}
$$

siendo $\mathrm{r}$ la velocidad de degradación fotocatalítica, $\mathrm{k}_{\mathrm{r}}$ la constante cinética de la reacción, $\mathrm{K}$ la constante de adsorción del contaminante sobre la superficie del catalizador y $\mathrm{C}_{0}$ la concentración inicial de sustrato.
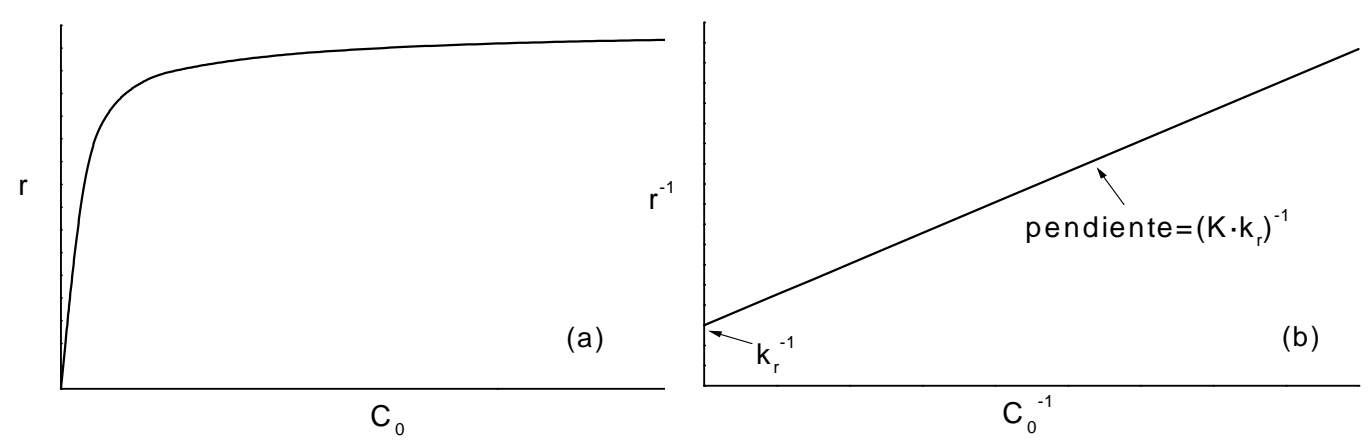

Fig. 5 Cinética de Langmuir-Hinshelwood. Velocidad de reacción en función de la concentración inicial (a) y ecuación L-H linealizada (b). 


\section{Radiación incidente}

Las reacciones de fotocatálisis pueden optimizarse aumentando el flujo fotónico que incide sobre el sistema, siempre y cuando el catalizador sea capaz de aprovecharlo.

Las investigaciones sobre este tema apuntan que la velocidad de reacción depende de la intensidad de la radiación inicialmente de forma lineal [Ollis, 1991; Herrmann, 1995].

A media que aumenta la intensidad la dependencia cambia a la raíz cuadrada de la intensidad, y si sigue aumentando llega un momento en el que la velocidad de reacción se hace constante, independientemente de la cantidad de fotones que entran en el reactor. Esto puede deberse a varias causas como:

- El defecto de moléculas captadoras de electrones $\left(\mathrm{O}_{2}\right)$.

- Las moléculas de sustrato a degradar y/o las moléculas de productos intermedios ya producidos saturen los centros activos superficiales del catalizador.

- Que, por otros motivos, las moléculas de sustrato no se acerquen lo suficiente al catalizador para ser oxidadas, etc.

Estos problemas pueden evitarse si se optimiza la superficie de catalizador expuesto, si se aumenta la presión parcial de oxígeno o se añaden otros captadores de electrones $\left(\mathrm{H}_{2} \mathrm{O}_{2}, \mathrm{~S}_{2} \mathrm{O}_{8}{ }^{2-}\right.$, etc. $)$, si se aumenta la velocidad de agitación favoreciendo un régimen de flujo turbulento en el reactor $\mathrm{y}$, por tanto, un mayor contacto entre el medio acuoso y el catalizador, etc.

\section{Temperatura}

La activación fotónica del catalizador no requiere aporte de temperatura, ya que la energía térmica no produce excitación del catalizador. A temperaturas especialmente bajas $\left(-40^{\circ} \mathrm{C}-0^{\circ} \mathrm{C}\right)$ y altas $(\mathrm{T}>70$ $80^{\circ} \mathrm{C}$ ), la actividad del proceso de fotocatálisis decrece para distintos tipos de reacciones fotocatalíticas.

Este comportamiento puede explicarse fácilmente dentro del marco de la cinética de LangmuirHinshelwood anteriormente descrito. Pero además, a ese rango de temperaturas se favorece la adsorción de los productos finales de reacción que bloquean la actividad del catalizador.

Por el contrario, cuando la temperatura del proceso aumenta por encima de $\operatorname{los} 80^{\circ} \mathrm{C}$, la adsorción de los reactivos está claramente desfavorecida siendo éste el factor limitante de la reacción. Además de todas estas limitaciones, otras consideraciones desde el punto de vista del diseño de un reactor de fotocatálisis deben tenerse en cuenta.

A temperaturas elevadas los materiales que constituyen el reactor sufren un deterioro considerable y la cantidad de oxígeno disuelto en agua disminuye. Por ello, se ha determinado que la temperatura óptima se encuentra entre $20^{\circ} \mathrm{C}$ y 80 ${ }^{\circ} \mathrm{C}$ [Blanco y Malato, 2001], lo cual facilita y economiza el diseño y funcionamiento de un reactor de fotocatálisis solar.

\section{Disposición del catalizador}

Un aspecto fundamental que determina la eficiencia del proceso fotocatalítico es la disposición del catalizador en el reactor. Existen dos tendencias en la investigación actual sobre este tema. Una es utilizarlo en suspensión acuosa y otra es fijarlo sobre un soporte de modo que el agua a tratar esté en contacto con él y en presencia de radiación UV adecuada se produzca la reacción fotocatalítica (Fig. 6 y 7).

Este segundo modo presenta frente al primero la ventaja de que no hay que eliminar el catalizador del agua una vez terminado el proceso de fotocatálisis.

Sin embargo, las eficiencias fotocatalíticas de sendas configuraciones son, en general, muy diferentes.

Normalmente, se obtienen procesos más eficaces de degradación con las suspensiones de $\mathrm{TiO}_{2}$ como han encontrado muchos autores de este campo; sirva de ejemplo la degradación de Benzamida con $\mathrm{TiO}_{2}$ fijado sobre fibra de vidrio en comparación con una suspensión de $\mathrm{TiO}_{2}$ Degussa-P25 obtenida por Robert et al. [Robert y col., 1999].

Esta eficiencia depende mucho del reactor y del tipo de compuesto. 

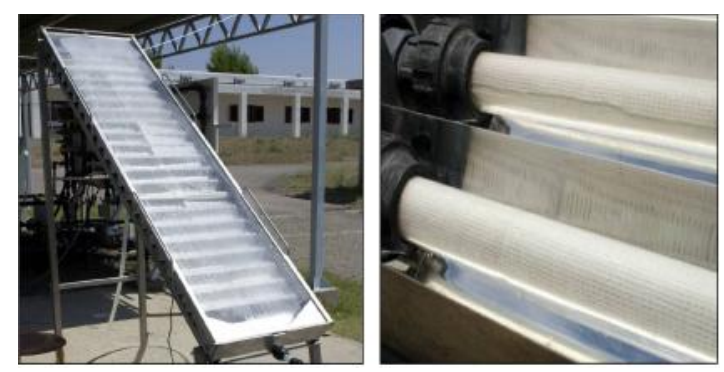

Fig.6 $\mathrm{TiO}_{2}$ fijado sobre papel en un reactor de escalera (izda.). $\mathrm{TiO}_{2}$ fijado sobre papel en un reactor tubural con concentración tipo CPC (Colector Parabólico Compuesto) (dcha.).

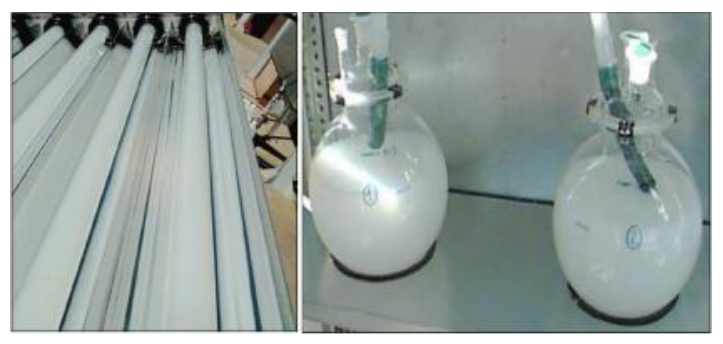

Fig.7 $\mathrm{TiO}_{2}$ en suspensión acuosa en un reactor tubular tipo CPC (izda) y en depósitos (dcha.).

\section{Caracterización coloidal de suspensiones de $\mathrm{TiO}_{2}$ en medio acuoso}

Los iones determinantes de potencial del $\mathrm{TiO}_{2}$ son los $\mathrm{OH}^{-}$y $\mathrm{H}^{+}$del agua. El punto de carga nula (PZC, Point of Zero Charge) de las partículas de dióxido de titanio en suspensión coloidal se define como el $\mathrm{pH}$ del medio para el cual la carga total libre de la superficie de las mismas es nula.

Su punto isoeléctrico (IEP, Isoelectric Point) es el $\mathrm{pH}$ para el cual la carga superficial se anula en presencia de los iones de cierto electrolito. Mediante técnicas de electroforesis se han determinado los valores de PZC y de IEP presencia de un electrolito, $\mathrm{NaCl}$, que proporciona iones muy comunes, siendo éstos igual a $\mathrm{pH} 7$ [Fernández-Ibáñez y col., 2000; Fernández-Ibáñez y col., 2003]. Así mismo, el comportamiento de las partículas en suspensión desde el punto de vista coloidal, a distintos $\mathrm{pHs}$ y concentraciones de electrolito, se analiza mediante medidas de tamaño medio de partícula o diámetro hidrodinámico obtenido por espectroscopía de fotocorrelación.
El comportamiento de las medidas de movilidad electroforética se muestra, junto con los ajustes a una curva de ajuste de tipo hiperbólico, en la figura 8 (arriba). La figura 8 (abajo) además presenta el comportamiento del tamaño medio de partícula frente al $\mathrm{pH}$ a concentraciones diferentes de $\mathrm{NaCl}$.

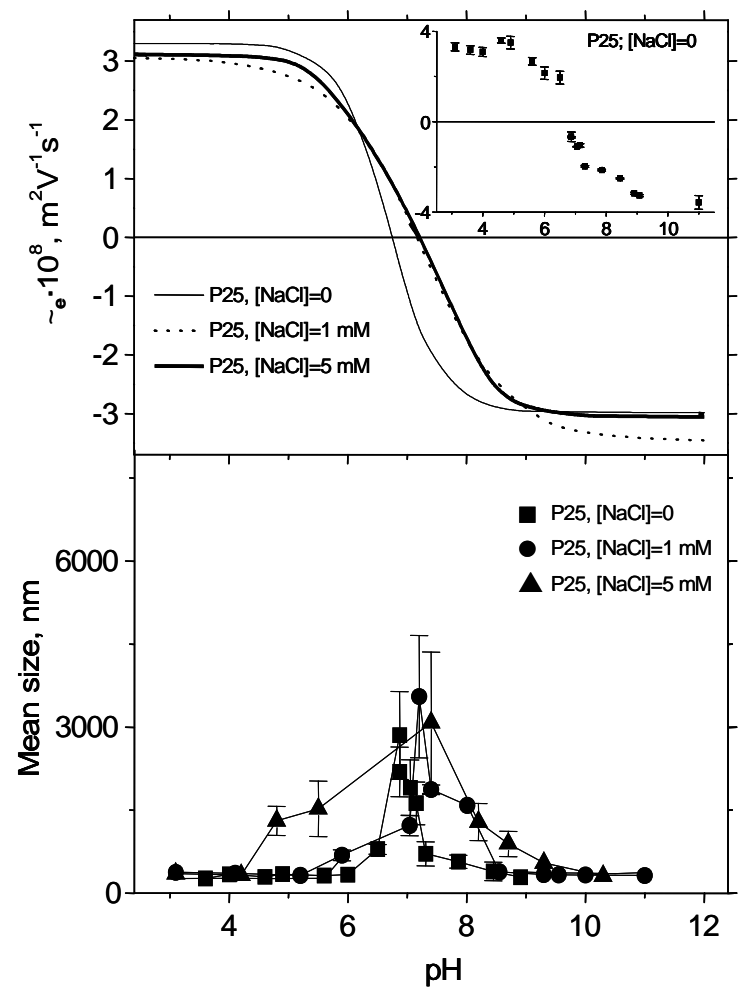

Fig. $8 \quad$ Ajuste de la movilidad electroforética (arriba) y tamaño medio de particular (abajo) frente al $\mathrm{pH}$. P25-TiO $(0.2 \mathrm{~g}$ L-1). Figura interior: datos experimentales de movilidad electroforética para $[\mathrm{NaCl}]=0$ [Fernández-Ibáñez y col., 2003].

Los resultados aquí presentados reflejan la clara y lógica evidencia de las características de las suspensiones de $\mathrm{TiO}_{2}$. En el PZC y en su IEP las partículas se encuentran sin carga eléctrica superficial, lo que impide que exista una interacción repulsiva entre ellas que impida su agregación a medida que éstas se acercan unas a otras como consecuencia de su movimiento dentro del fluido. Por ello, se produce una agregación del sistema a valores de $\mathrm{pH}$ muy cercanos a 7, lo cual viene dado por el elevado tamaño medio de partícula observado a esos pHs. Del mismo modo, 
se obtienen partículas con carga y de tamaño medio mucho más pequeño en las regiones de $\mathrm{pH}$ suficientemente alejadas del PZC y del IEP, ya que no se produce la agregación anterior (estabilidad coloidal [Hunter, 1993]).

\section{Separación del catalizador}

Los resultados del estudio anterior se han aplicado a un sistema de sedimentación rápida de partículas de fotocatalizador $\left(\mathrm{TiO}_{2}\right)$ basado en su elevada densidad de masa $\left(3.5 \mathrm{~g} / \mathrm{cm}^{3}\right)$ y en el conocimiento de sus condiciones de estabilidad e inestabilidad coloidal [Fernández-Ibáñez y col., 2003]. El esquema del concepto de trabajo de tal método y su aplicación a una planta piloto acoplada a una planta de tratamiento de aguas mediante fotocatálisis solar viene dado en la figura 9, arriba y abajo respectivamente. Éste ha sido patentado [CIEMAT, 2001] y probado en la primera planta de fotocatálisis solar de Europa construida a nivel industrial experimental [Malato y col., 2002].

Tras una experiencia de fotocatálisis solar de degradación de un orgánico disuelto, se retira el agua con el dióxido de titanio y los iones inorgánicos, procedentes de la degradación, de los colectores solares y se almacena en un tanque de sedimentación. Ahí el pH se ajusta hasta alcanzar el PZC y se observa la sedimentación rápida de las partículas de $\mathrm{TiO}_{2}$.

El seguimiento de la pérdida de catalizador a $\mathrm{pH} 7$ en el sobrenadante del agua, en comparación con la suspensión a $\mathrm{pH} 3$, se realiza con medidas de absorbancia a $800 \mathrm{~nm}$ de longitud de onda (Fig.10). Una vez el contenido sólido de la suspensión se encuentra en el fondo del tanque, se extrae el agua del sobrenadante con ayuda de una bomba y se pasa a través del sistema de membranas de ultrafiltración de la planta. El agua resultante es un agua limpia de $\mathrm{TiO}_{2}$ y de contaminantes (Fig. 10 interior).
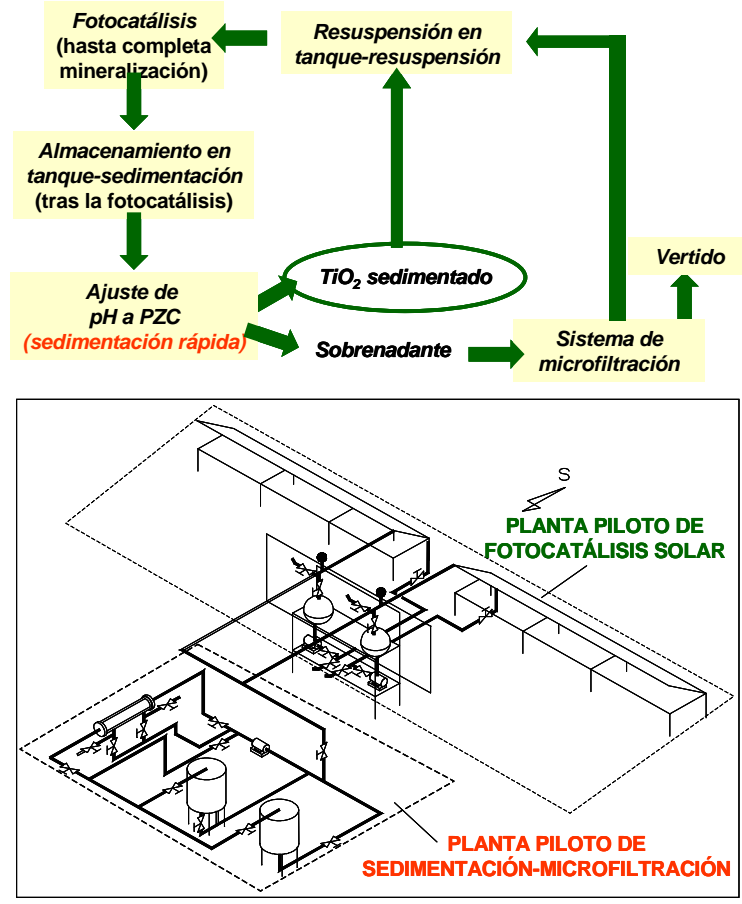

Fig. 9 Esquema de método de sedimentación (arriba) y planta piloto de sedimentación acoplada a una planta piloto de fotocatálisis (abajo).

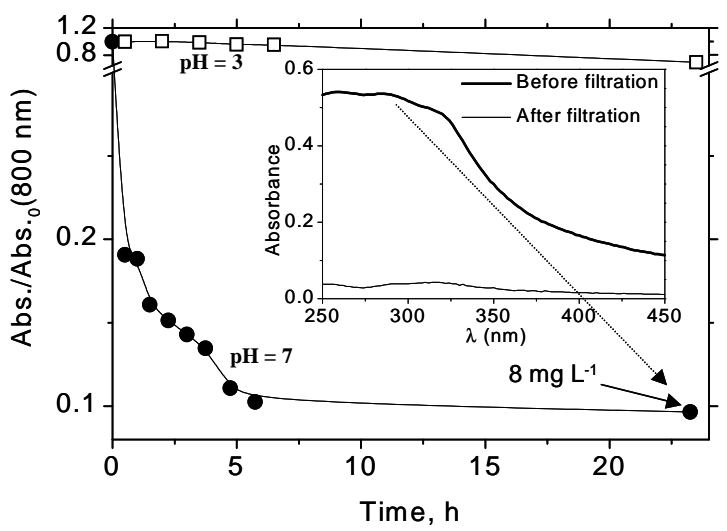

Fig. 10 Absorbancia de la suspension de $\mathrm{TiO}_{2} \mathrm{P}$ 25 (Concentración inicial $=200 \mathrm{mg} / \mathrm{L})$ durante un test de sedimentación a escala piloto $(384 \mathrm{~L})$. En el interior se muestra un espectro de absorbancia del agua después de la sedimentación $\left(\left[\mathrm{TiO}_{2}\right] \sim 8 \mathrm{mg} / \mathrm{L}\right)$ y después de la ultrafiltración. 


\section{REFERENCIAS}

1. Blanco, J.; Malato, S., "Tecnología de Fotocatálisis Solar". Cuadernos Monográficos 31. Instituto de Estudios Almerienses de la Diputación de Almería. Almería, España, 1996.

2. Schertenleib, R., Gujer, W.; EAWAG News, 48, 3-5, Septiembre 2000.

3. Andreozzi, R., Caprio, V.; Insola, A.; Marotta, R.; "Advanced Oxidation Processes (AOP) for Water Purification and Recovery". Catalysis Today, 53, 51-59, (1999).

4. Bahnemann, D.W.; Bockelmann, D.; Goslich, R.; Hilgendorff, M.; Weichgrebe, D; "Photocatalytic Detoxification: Novel Catalyst, Mechanism and Solar Applications". Photocatalytic Purification and Treatment of Water and Air, D. Ollis and H. Al-Ekabi (eds.), Elsevier Sciencie Publishers, 301-319, Amsterdam (1993).

5. Bahnemann, D.W.; Cunningham, J.; Fox, M.A.; Pelizzetti, E.; Serpone, N.; "Photocatalytic Detoxification of Polluted Aquifers: Novel Catalyst and Solar Applications". Aquatic and Surface Photochemistry. G.R. Helz, R.G. Zepp and D.G. Crosby (eds.). Lewis Publishers. 349368, (1994a).

6. Bahnemann, D.W.; Cunningham, J.; Fox, M. A.; Pelizzetti, E.; Serpone, N.; "Photocatalytic Treatment of Water". Aquatic and Surface Photochemistry. G.R. Helz, R.G. Zepp and D.G. Crosby (eds.). Lewis Publishers, 261316, (1994b).

7. Bauer, R.; "Applicability of Solar Irradiation for Photochemical Wastewater Treatment". Chemosphere, 29(6), 1225-1233, (1994).

8. Bauer, R.; Waldner, G.; Fallmann, H.; Hager, S.; Klare, M.; Krutzler, T.; Malato, S.; Maletzky, P.; "The Photo-Fenton Reaction and the $\mathrm{TiO}_{2} / \mathrm{UV}$ Process for Waste Water Treatment - Novel Developments". Catalysis Today, 53, 131-144, (1999).

9. Blanco, J.; Malato, S.; "Solar Detoxification". UNESCO, Natural Sciences, WORLD SOLAR PROGRAMME 1996-2005 (2001).

10. Bockelmann, D.; Weichgrebe, D.; Goslich, R.; Bahnemann, D.; "Concentrating versus non-Concentrating Reactors for Solar Water Detoxification". Sol. Energ. Mat. Sol. Cells., 38, 441-451, (1995).
11. Curcó, D.; Malato, S.; Blanco, J.; Giménez, J.; "Photocatalysis and Radiation Adsorcion in a Solar Plant". Sol. En. Mat. Sol. Cells, 44, 199 (1996).

12. Freundenhammer, H.; Bahnemann, D.; Bousselmi, L.; Geissen, S.U.; Ghrabi, A.; Saleh, F.; Si-Salah, A.; Siemon, U.; Vogelpohl, A.; "Detoxification and Recycling of Wastewater by Solar-Catalytic Treatment". Water Sci. Technol., 35(4), 149-156, (1997).

13. Goslich, R.; Dillert, R.; Bahnemann, D.; "Solar Water Treatment. Principles and Reactors". Wat. Sci. Tech., 35(4), 137-148, (1997).

14. Giménez, J.; Curcó, D.; Marco, P.; "Reactor Modelling in the Photocatalytic Oxidation of Wastewater". Water Sci. Tech., 35(4), 207213, (1997).

15. Giménez, J.; Curcó, D.; Queral, M.A.; "Photocatalytic Treatment of Phenol and 2,4dichlorophenol in a Solar Plant in the Way to Scaling-up". Catalysis Today, 54, 229-244, (1999).

16. Herrmann, J.M.; Disdier, J.; Pichat, P.; Malato, S.; Blanco, J.; " $\mathrm{TiO}_{2}$-based Solar Photocatalytic Detoxification of Water Containing Organic Pollutants. Case Studies of 2,4-dichlorophenoxyacetic acid (2,4-D) and of Benzofuran”. Appl. Catal. B: Environ., 17, 1523, (1998).

17. Herrmann, J.M.; Matos, J.; Disdier, J; Guillard, C.; Laine, J.; Malato, S.; Blanco, J.; "Solar Photocatalytic Degradation of 4Chlorophenol Using the Synergistic Effect between Titania and Activated Carbon in Aqueous Suspension". Catalysis Today, 54, 255-265, (1999).

18. Malato, S.; Blanco, J.; Herrmann, J.M.; (Eds.) "Solar Catalysis for Water Decontamination". Catalysis Today, 54 (2-3), (1999).

19. Malato, S.; Blanco, J.; Vidal, A.; Richter, C.; "Photocatalysis with Solar Energy at a Pilotplant Scale: an Overview". Appl. Catal. B: Environ., 37, 1-15, (2002).

20. Marques, P.A.; Rosa, M.F.; Mendes, F.; Collares Pereira, M.; Blanco, J.; Malato, S.; "Wastewater Detoxification of Organic and Inorganic Toxic Compounds with Solar Collectors". Desalination, 108, 213-220, (1996).

21. Minero, C.; "Photocatalytic Transformation of Hydrocarbons at the Sea Water / Air Surfase 
under Solar Irradiation". Marine Chem., 58, 361-372, (1997).

22. Zhang, T.; Oyama, T.; Horikoshi, S.; Zhao, J.; Hidaka, H.; Serpone, N.; "Assessment and Influence of Operational Parameters on the $\mathrm{TiO}_{2}$ Photocatalytic Degradation of Sodium Benzene Sulfonate under Highly Concentrated Solar Light Illumination". Solar Energy, 71(35), 305-313, (2001).

23. Blake, D.M.; (a) "Bibliography of Work on the Photocatalytic Removal of Hazardous Compounds from Water and Air". (b) "Update Number 1 To June 1995". (c) "Update Number 2 To October 1997". (d) "Update Number 3 To January 1999". (e) "Update Number 4 to October 2001". National Technical Information Service, US Dep. of Commerce, Springfield, VA22161, USA, 1994, 1995, 1997, 1999 y 2001.

24. Guillard, C.; Disdier, J.; Herrmann, J.M.; Lehaut, C.; Chopin, T.; Malato, S.; Blanco, J.; "Comparison of Various Titania Samples of Industrial Origin in the Solar Photocatalytic Detoxification of Water Containing 4Chlorophenol". Catalysis Today, 54, 217-228, (1999).

25. Prousek, J.; "Advanced Oxidation Processes for Water Treatment. Photochemical Processes". Chem. Listy, 90, 307-315, (1996).

26. Serpone, N.; Tercian, R.; Minero, C.; Pelizzetti, E.; "Heterogeneous Photocatalyzed Oxidation of Phenol, Cresols, and Fluorphenols in $\mathrm{TiO}_{2}$ Aqueous Suspension". Adv. Chem. Ser., 238, 281-314, (1993).

27. Tomlin, C.D.S.; The Pesticide Manual, a World Compendium. 11 ${ }^{\text {th }}$ Edition. British Crop Protection Council. Croydon, UK, (1998).

28. Fernández-Ibáñez, P.; Blanco, J.; Malato, S.; de las Nieves, F.J.; "Application of the colloidal stability of $\mathrm{TiO}_{2}$ particles for recovery and reuse in solar photocatalysis". $J$. Water Research, 37, 3180 (2003).

29. Hulstrom, R.; Bird, R.; Riordan, C.; "Spectral Solar Irradiance Data Sets for Selected Terrestial Conditions". Solar Energy Research Institute, 15, 365-391, (1985).

30. Ollis, D.F.; "Solar-Assisted Photocatalysis for Water Purification: Issues, Data, Questions". Photochemical Conversion and Storage of Solar Energy, Kluwer Academic Publishers, 593-622, (1991).
31. Robert, D.; ,Piscopo, A.; Heintz, O.; Weber, J. V.; "Photocatalytic detoxification with $\mathrm{TiO}_{2}$ supported on glass-fibre by using artificial and natural light" Catalysis Today, 54, 291296 (1999).

32. Fernández-Ibáñez, P.; de las Nieves, F.J.; Malato, S.; "Titanium Dioxide/Electrolyte Solution Interface: Electron Transfer Phenomena". J. Colloid Interface Sci., 227, 510 (2000).

33. Hunter, R.J; Introduction to Modern Colloid Science. Oxford University Press (1993).

34. CIEMAT, "Method of sedimentation of colloidal semiconductor particles". European Patent EP9902508 (2001).

35. Malato, S.; Blanco, J.; Vidal, A.; Fernández, P.; Cáceres, J.; Trincado, P.; Oliveira, J. C.; Vincent, M.; "New large solar photocatalytic plant: set-up and preliminary results". Chemosphere, 47 (3), 235-240 (2002). 